Natural shear wave imaging using vocal tract vibrations

\title{
Natural shear wave imaging using vocal tract vibrations: introducing Vocal Passive Elastography (V-PE) to thyroid elasticity mapping
}

\author{
Steve Beuve, ${ }^{1, a)}$ Samuel Callé, ${ }^{2}$ Elise Khoury, ${ }^{1}$ Emmanuel Gilles Simon, ${ }^{3,4}$ and Jean-Pierre Remenieras ${ }^{1}$ \\ ${ }^{1)}$ UMR 1253, iBrain, Université de Tours, Inserm, 37000 Tours, France. \\ ${ }^{2)}$ GREMAN UMR 7347, Université de Tours, CNRS, INSA Centre Val de Loire, 37200 Tours, \\ France. \\ ${ }^{3)}$ Department of Obstetrics, Gynecology and Fetal Medicine, CHU Dijon Bourgogne, 21000 Dijon, \\ France \\ ${ }^{4)}$ UBFC, Université Bourgogne Franche-Comté, 25000 Besançon, France \\ (Dated: 18 November 2020)
}

We present a shear wave elastography approach for thyroid characterization that is inspired by passive elastography which extracts elasticity from the natural vibrations in living tissues that are caused by cardiac motion, blood pulsatility and muscle activity. On thyroid, this physiological noise is mainly due to the carotid pulsation which is in the $1-10 \mathrm{~Hz}$ bandwidth and is located right next to the gland. In order to decrease the shear wavelength and increase the signal to noise ratio, we propose to create in the thyroid a complex shear wave field by using natural vocal tract vibrations. The nature of the sound can be easily modified to be narrow or broad band, with small or large amplitude. Using correlation based algorithm and a sound sustained at $150 \mathrm{~Hz}$, we have developed an innovative technique using ultrasound, allowing us to compute a 2D shear wave velocity map, superposed onto a B-mode ultrasound image of a volunteer's thyroid. Using our Vocal Passive Elastography (V-PE) method, shear wave velocity was measured at every point within a mask surrounding the thyroid with a pixel resolution of $150 \times 150 \mu \mathrm{m}^{2}$. The mean shear wave speed value measured is $3.2 \mathrm{~m} / \mathrm{s}$, taking its value from $0.7 \mathrm{~m} / \mathrm{s}$ to $8.8 \mathrm{~m} / \mathrm{s}$. The values obtained were in good agreement with comparative Shear Wave Elastography (SWE) measurements.

Thyroid nodular disease is one of the most widespread endocrine disorders ${ }^{1}$. For example, papillary thyroid cancers account for about $80 \%$ of all thyroid disease cases. Furthermore, children and women aged 20 to 55 are most commonly affected $^{2}$. The thyroid is a gland located in the throat surrounding the trachea. Its function is to secrete hormones essential for the metabolism and growth of the body.

Although fine needle aspiration guided with ultrasound (US) examination is commonly used to detect malignant tumors, only $5 \%$ of thyroid cancers are detectable with this technique ${ }^{1}$. Elastography seems to be a good candidate for detecting cancerous nodules since stiffness is one of the most important parameters of nodular characterization. Accordingly, a nodule whose Young's modulus exceeds $65 \mathrm{kPa}$ is usually considered suspicious ${ }^{1}$. More than 100 articles concerned with the detection of thyroid disease using various elastographic methods (mainly static, ARFI and $\mathrm{SWE}^{3}$ ) have been published since $2005^{4-6}$. One report even took advantage of the waves generated by carotid movements for passive applications ${ }^{7}$.

Our approach is premised in this context; the challenge is to identify malignant follicular tumors that currently escape detection by the association of ultrasound examination and fine needle aspiration biopsy. We have designed an experiment that takes advantage of a shear wave acoustic field generated naturally by the human voice to measure the elasticity of thyroid tissue. Since the trachea is situated near to the thy-

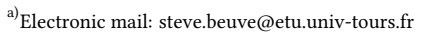

roid, shear wave SNR is relatively high. To track shear waves, an ultrasound scanner set in ultrafast mode is used. Correlation algorithms based on Time Reversal methods ${ }^{8}$, initially used in seismology ${ }^{9-12}$ are adapted to physiological noise to determine shear wave speeds, as they propagate through the thyroid.

The volunteer produces and maintains a monotonous vocal tone [ə]. To assist the volunteer with the maintenance of the required tone, a loudspeaker plays a $150 \mathrm{~Hz}$ sound. As shown in Fig. 1 and 2, the thyroid is right next to the trachea that will vibrate during singing. An acoustic field crossing the gland is expected. Unlike most current passive elastography techniques, we chose to generate monochromatic sounds thereby ensuring that the waves induced in the thyroid came from the voice. The resultant shear waves were tracked at an ultrafast frame rate using an ultrasound system. Finally, shear wave speed were computed using passive elastography methods and mapped on the thyroid image to localize any abnormally stiff regions. This is the principle of Vocal Passive Elastography (V-PE).

To obtain an editable movie of thyroid particle motion, 400 images are acquired at high frame rate using a programmable Verasonics Vantage ${ }^{\mathrm{TM}}$ research ultrasound system equipped with a linear L11-4v probe operating at $10 \mathrm{MHz}$. The probe was placed horizontally against the surface of the neck (Fig. 1), thus allowing measurement of the shear wave speed at the cross section (horizontally)

Each frame comprised 5 plane waves from $-3^{\circ}$ to $3^{\circ}$ with $1.5^{\circ}$ incremental steps, separated by $66 \mu \mathrm{m}$ and designed to reach a Pulse Repetition Frequency (PRF) of 3000 frames per second during $132 \mathrm{~ms}$. The ultrasonic raw data from the Verasonics scanner is sampled at 


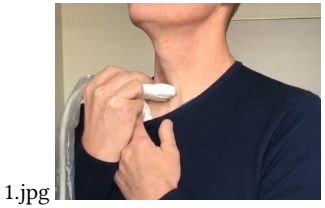

FIG. 1. A volunteer holding the probe horizontally against the surface of the neck during cross sectional measurement. Written informed consent was obtained from the volunteer.

8 points per US wavelength. To reconstruct the images, home made Delay And Sum (DAS) beamforming algorithm is applied to the raw data with an $\mathrm{F}$ number equal to one. To get nearer to the axial resolution $(d z \approx$ pitch $/ 10=30 \mu \mathrm{m})$, latera sampling was divided by $2(d x=$ pitch $/ 2 \approx 5 d z=150 \mu \mathrm{m})$ for the DAS algorithm. Data was rendered complex IQ fromat by applying the Hilbert transform on the axial dimension. Local particle motion was then observed by watching the brightness mode image as a function of time and axial moving averaging was used to make pixels square $150 \times 150 \mu \mathrm{m}^{2}$. As expected, the highest motion magnitude was localized around the trachea (Fig. 2).

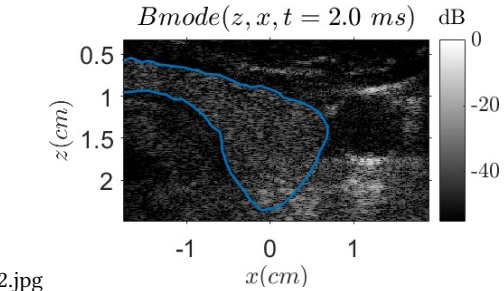

FIG. 2. Brightness mode image in the cross section with the thyroid located between the trachea (at the bottom-left of the image), carotid artery (the black hole on the right) and a mask surrounding the thyroid (in blue) where the local wave speed is to be estimated.

The tissue particle velocity was calculated using one sample shifted autocorrelation of IQ data ${ }^{13}$ while a low pass filter was used to reduce phase noise and permit tracking of the axial component of the shear waves propagating into the thyroid.

To study the characteristics of the waves produced by the voice (Fig. 3) the particle velocity data from the thyroid were extracted with a manually drawn mask and plotted into the B-mode image. Focusing our attention on the left side of the images in Fig. 3, the waves appear directional, coming as they do from the left side and propagating horizontally. The acoustic field on the bottom right side seems complex with waves coming from different directions. The resultan field is therefore more complex on the right compared with the region of interest on the left.

The maximum particle velocity is about $\mathbf{v}_{m a x}=10 \mathrm{~mm} / \mathrm{s}$ at $f_{\text {ext }}=150 \mathrm{~Hz}$. The particle tissue displacement induced in the gland, using the voice, is then $\mu_{\max }=\mathbf{v}_{\max } / f_{\text {ext }}=$
$(10 \mathrm{~mm} / \mathrm{s}) /(150 \mathrm{~Hz})=10 \mu \mathrm{m}$. This displacement is easily determined with our system since the noise level is of the order of half a micron.

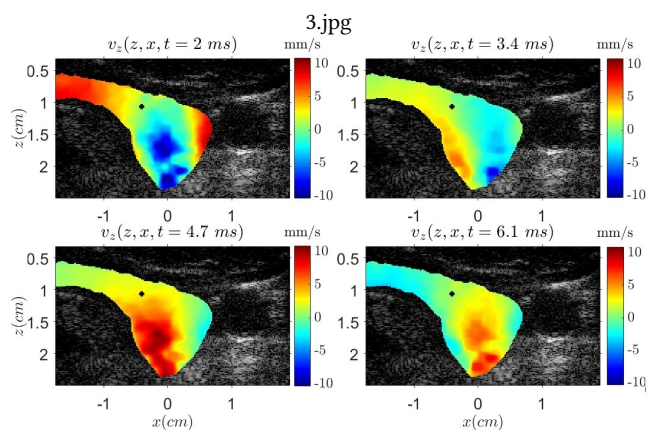

FIG. 3. Particle velocity map $v_{\mathrm{z}}(z, x)$ at different times $t=2.0,3.4$, 4.7 and $6.1 \mathrm{~ms}$ between which the $\mathrm{S}$-wave has traveled around three quarters of a wavelength in the thyroid and overlaid on the brightness mode image.

By selecting a point in the region of interest we can plot the particle velocity and its Fourier transform. That allows us to see the result of the monotonous singing (Fig. 4). The magnitude of the Fourier transform shows a peak at $f_{\text {ext }}=$ $150 \mathrm{~Hz}$ i.e. the frequency of the vocal note emitted by the volunteer.
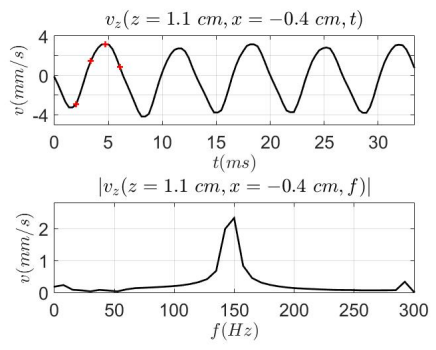

4.jpg

FIG. 4. Temporal representation of 5 periods of the axial particle velocity $\mathbf{v}_{\mathrm{z}}(t)$ at a nominal point $(z=10 \mathrm{~cm}, x=-0.4 \mathrm{~cm})$ and its Fourier transform magnitude $\left|\mathbf{v}_{\mathbf{z}}(f)\right|$.

A small second harmonic was observed at $300 \mathrm{~Hz}(\mathrm{n}=2)$ on Fig. 4. One can wonder if this harmonics was created by a cumulative non-linear effect during the shear wave propagation. This even harmonic was also reported by Giammarinaro ${ }^{14}$ for studies on the non-linear plane shear waves induced by an acrylic plate as they propagated through a gelatin phantom. But looking the shear wave non-linear propagation equation, we note that it contains a non-linear term which is cubic instead of quadratic for the compressional wave. Thus the cumulative nonlinear harmonics are 
odd $(2 \mathrm{n}+1) f_{\text {ext }}{ }^{15}$ for shear wave and the non-linear propagation is not the explanation of our spectrum on Fig. 4. This shear wave at $300 \mathrm{~Hz}$ may be due to the voice which is not strictly pure in frequency. The timbre is what makes a particular musical instrument or human voice have a different sound from another even when they play or sing the same note, and is directly linked to the amplitude of the harmonics. This second harmonic is then probably due to a linear propagation from the source at $300 \mathrm{~Hz}$.

To have a better understanding of the mechanism involved in the generation of shear wave through the organ, it would be necessary to model the source, the larynx, associated with the different resonators of the vocal track, the pharynx, the oral cavity, the labiodental cavity and, for some sounds, the nasopharynx and the nasal cavity. They have to be in a specific configuration to permit the emission of the [ə] sound at $150 \mathrm{~Hz}$ in our experiment. Badin et al. ${ }^{16}$ for example realized a biomechanical modeling of the organs of the vocal tract consisting of determining a certain number of basic properties and movements which make it possible to represent simply the complex movements of the articulators such as the jaw, the tongue, the veil of the palate and the lips. In first approximation, the vocal track could be compared to a resonant cavity in the form of an air-filled tube with a variable diameter.

The passive elastography algorithm seeks to solve the inverse problem by analyzing waves induced physiologically to find back medium elastic parameters. Recent studies have shown that passive elastographic methods improve with multiple active sources around the region of interest ${ }^{17}$. This ratio of spatio-temporal derivatives to autocorrelations Eq. (1) permits resolution of the dispersion equation ${ }^{18}$ in a manner equivalent to the time-reversal approach.

$$
\mathbf{c}_{s}(z, x)=\sqrt{\frac{\mathbf{a}_{\mathrm{TR}}}{\dot{\varepsilon}_{\mathrm{TR}}}}
$$

where $\mathbf{a}_{\mathrm{TR}}$ is the particle acceleration autocorrelation, $\dot{\varepsilon}_{\mathrm{TR}}$ the particle strain rate autocorrelation, and $c_{\mathrm{s}}$ the local shear wave group velocity. Derivatives are calculated by measur ing the slope of the linear regression in a sliding window ${ }^{19}$ using three adjacent points. The value chosen for the autocorrelation is that corresponding to the time of the collapse, i.e., the refocusing time in Catheline's theory ${ }^{20}$.

$$
\begin{gathered}
\mathbf{a}_{\mathrm{TR}}=C\left(\mathbf{a}_{\mathrm{z}}\right)(0)=-\omega^{2} C\left(\mathbf{v}_{\mathrm{z}}\right)(0) \\
\dot{\varepsilon}_{\mathrm{TR}}=C\left(\dot{\varepsilon}_{\mathrm{zx}}\right)(0)+C\left(\dot{\varepsilon}_{\mathrm{zz}}\right)(0)=-\mathbf{k}^{2} C\left(\mathbf{v}_{\mathrm{z}}\right)(0)
\end{gathered}
$$

where $\mathbf{v}_{\mathbf{z}}$ is the axial particle velocity, $\mathbf{k}^{2}=\mathbf{k}_{\mathbf{z}}{ }^{2}+\mathbf{k}_{\mathbf{x}}{ }^{2}$ the square wavelength and $C$ the autocorrelation operator at each point of the mesh encompassing the thyroid mask. This operation brings up the angular frequency $\omega$ Eq. (2a) and the wavenumber $k$ Eq. (2b).

An alternative to the Fourier transform approach to measure the frequency $f$ of the signal within the thyroid is the autocorrelation ratio of the particle acceleration $a_{\mathrm{TR}}$ and velocity $v_{\mathrm{TR}}$ linked by the pulsation $\omega$ through the equation:

$$
\mathbf{f}(z, x)=\frac{1}{2 \pi} \sqrt{\frac{\mathbf{a}_{\mathrm{TR}}}{\mathbf{v}_{\mathrm{TR}}}}
$$

The time frequency is calculated with the autocorrelation in the 2D plane of the thyroid using equation Eq. (3). In this way, the entire $f$ map is much closer to the value targeted by the volunteer $f_{\text {ext }}=150 \mathrm{~Hz}$ (Fig. 5).

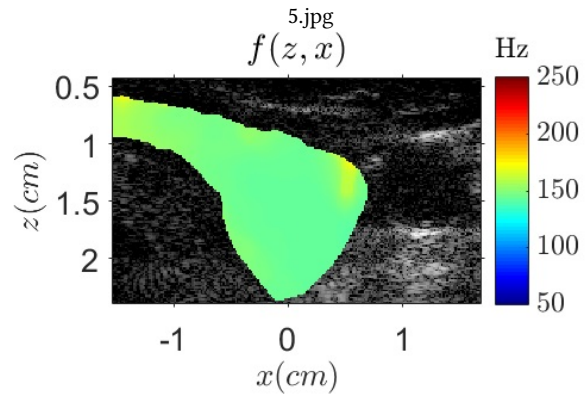

FIG. 5. 2D frequency $f$ of the particle velocity $v_{z}$ in the thyroid overlaid on the corresponding anatomical brightness mode image.

The time frequency statistical measurement data for the thyroid are listed in the Table I :

TABLE I. Local time frequency values of the acoustic waves produced by vocal tract at all points of the volunteer's thyroid using the passive elastography technique. Mean value, minimum, maximum and standard deviation were computed.

\begin{tabular}{cc}
\hline \hline Statistics & Frequency \\
\hline $\operatorname{mean}(f)$ & $148 \mathrm{~Hz}$ \\
$\min (f)$ & $143 \mathrm{~Hz}$ \\
$\max (f)$ & $177 \mathrm{~Hz}$ \\
$\operatorname{std}(f)$ & $4.02 \mathrm{~Hz}$ \\
$\operatorname{rmse}\left(f-f_{\text {ext }}\right)$ & $4.56 \mathrm{~Hz}$ \\
\hline \hline
\end{tabular}

Then the autocorrelation ratio of the particle acceleration and strain rate is calculated in all points located in the mask drawn around the thyroid, thanks to Eq. (1), to map the shear wave speed into it (Fig. 6) 


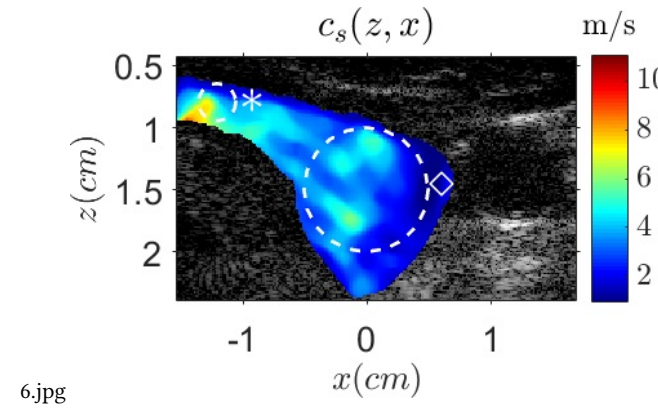

FIG. 6. 2D Shear wave speed $c_{\mathrm{S}}$ in the thyroid measured with the VPE method and overlaid on the anatomical brightness mode image with two Q-box ${ }^{\mathrm{TM}}$ equivalents $\diamond$ and $*$.

To obtain a comparative element, the shear wave velocity was measured by SWE using an AIXplorer ${ }^{\circledR}$ US scanner (SuperSonic Imagine, 13857, Aix-en-Provence, France) equipped with a linear SL10-2 probe ${ }^{21}$. To validate our V-PE algorithm, we created an equivalent $\mathrm{Q}-$ box $^{\mathrm{TM}}$ using tools introduced by Supersonic Imagine and compared the data obtained with Q-box ${ }^{\mathrm{TM}}$ from the AIXplorer ${ }^{\circledR}$ for the same area of the volunteer's thyroid. The Q-box ${ }^{\mathrm{TM}}$ equivalent areas were modeled on those used with the SSI model Q-box ${ }^{\mathrm{TM}}$ regardless of whether they were perfectly identical.

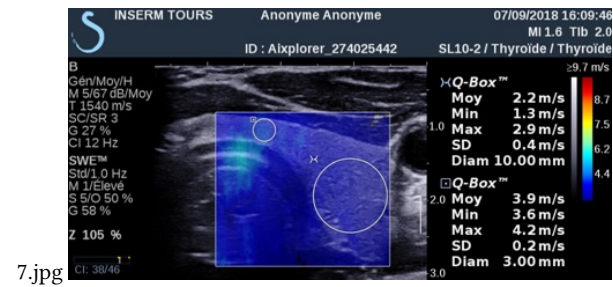

FIG. 7. SuperSonic Imagine 2D map of the shear wave velocity in the volunteer's thyroid (Fig. 1) measured using the SWE technique. The mean value, minimum, maximum and standard deviation of the local shear wave velocity were computed for areas denoted $\square$ (upper left corner of the ROI) and $x$ (lower right corner of the ROI) $Q$ box ${ }^{\mathrm{TM}}$

The results presented in Table II exhibit the closeness in elasticity that is also noticeably higher for regions on the left for both V-PE and SWE techniques.
TABLE II. Speed values of the acoustic waves propagating through the volunteer's thyroid measured using the V-PE technique in the transverse section for a monochromatic excitation signal. Two areas, $\diamond$ and $*$, were drawn and values extracted with the aim of comparing the resultant V-PE values with those measured using the SWE method. Mean value, minimum, maximum, and standard deviation are computed for areas $\diamond($ Diameter $=10 \mathrm{~mm})$ and $*($ Diameter $=3 \mathrm{~mm}$ ).

\begin{tabular}{ccc}
\hline \hline Q-box ${ }^{\mathrm{TM}}$ equivalent & $\diamond$ & $*$ \\
\hline $\operatorname{mean}\left(c_{\mathrm{s}}\right)$ & $3.3 \mathrm{~m} / \mathrm{s}$ & $4.5 \mathrm{~m} / \mathrm{s}$ \\
$\min \left(c_{\mathrm{s}}\right)$ & $0.8 \mathrm{~m} / \mathrm{s}$ & $1.3 \mathrm{~m} / \mathrm{s}$ \\
$\max \left(c_{\mathrm{s}}\right)$ & $5.9 \mathrm{~m} / \mathrm{s}$ & $7.6 \mathrm{~m} / \mathrm{s}$ \\
$\operatorname{std}\left(c_{\mathrm{s}}\right)$ & $1.1 \mathrm{~m} / \mathrm{s}$ & $1.8 \mathrm{~m} / \mathrm{s}$ \\
Diameter & $10 \mathrm{~mm}$ & $3 \mathrm{~mm}$ \\
\hline \hline
\end{tabular}

For the V-PE method on the left side of the thyroid, the $\mathrm{S}$ waves are directional and propagates mainly horizontally along the positive $\mathrm{x}$ axis, with a large magnitude transverse displacement along the $\mathrm{z}$ axis. In this region, waves can be guided since the thyroid thickness is 6 times smaller than the shear wavelength $(\lambda$ $=(4.5 \mathrm{~m} / \mathrm{s}) /(150 \mathrm{~Hz})=3 \mathrm{~cm}=6 \times 0.5 \mathrm{~cm})$. The shear wavelength on the right side area is of the same order of magnitude than the gland size $(\lambda=(3.3 \mathrm{~m} / \mathrm{s}) /(150 \mathrm{~Hz})=2.2 \mathrm{~cm} \approx 2 \mathrm{~cm})$. In the case of guided waves, the wave speed depends on the thickness of the propagation medium ${ }^{22}$. So, if the mechanical properties of thyroid remain homogeneous in the imaging zone, we can measure different wave speed values between these two areas. Moreover, when we compare the results obtained using the V-PE and the SWE methods, we can point to a slight overestimation for results obtained with our technique compared to the SWE. In our opinion, the explanation of this overestimated celerity is due to the difference between the central frequencies of the shear wave sources. The waveguide effect induced a dispersion of the celerity with frequency ${ }^{23}$ and since the central frequencies of the two techniques are not the same, this could influence the measured speed of the dispersive wave. This dispersion may also come from the shear viscosity which increases the stiffness with frequency and also makes the wave faster with frequency. A solution to go further in the analysis could be to apply a shear wave spectroscopy analysis as it was done using SWE for the tendon $^{22}$, and to imagine a V-PE spectroscopy method with a broad band sound generated by complex vocalizations. This dispersion analysis is out of the scope of this letter but is a short-term perspective of this work.

There are several key advantages to Vocal Passive Elastography (V-PE). First, in sinusoidal steady-state shear wave excitation, the evolution of the wavefield pattern can be measured with any Doppler imaging platform and as a result, not necessary to have any synchronization or specialized platform with complex push or imaging sequences. Secondly, with the appropriate choice of the shear wave frequency, entire organs can be covered with waves providing large ROIs for analysis. For the thyroid, the $150 \mathrm{~Hz}$ frequency appears well-adapted, as it satisfies this criterion but we can imaging to induce a higher frequency to shorten the wavelength 
while meeting this criterion. The inverse problem based on the temporal analysis of the 2D spatial correlation naturally adapts to the complex nature of the wave field resulting from multiples sources. Hence the source condition can remain an unknown problem. This is a clear break from the SWE and vibration controlled transient elastography (VCTE) methods where the source condition has to be precisely controlled. Furthermore, from a superposition principle, multiple sources can induce a wavefield with a high signal-to-noise ratio, creating favorable conditions for estimating elasticity over a wide area. This constructive interaction is useful in brain applications, for example, where one may be interested in the induction of a wave field pattern with high SNR, using low-frequency vibration from voice.

Owing to its heterogeneity, the thyroid allows us to provide two mean values corresponding to two distinct zones, $c_{s}=4.5 \mathrm{~m} / \mathrm{s}$ on the left i.e. the thinner section, and $c_{s}=3.3 \mathrm{~m} / \mathrm{s}$ on the right i.e. the thickest section, both at frequency $f=$ $150 \mathrm{~Hz}$. However, some points contradict our expectation about the directivity of waves as the acoustic field induced by vocal tract is found to be directive, far from a diffuse field. The voice induces shear waves then the thyroid acts as a waveguide and propagates those laterally. Applying passive elastography computation allows for the mapping of the shear wave velocity on the thyroid image and delivers results in agreement with the SWE technique. Specifically, those values help us to mechanically characterize every point of the thyroid.

Further experiments to extend this work are underway, and our first concern is rooted in the realization that the more the wave field is complex; the more efficient is the passive elastography algorithm. So we plan to no longer restrict ourselves to a monochromatic signal but instead widen the bandwidth. This will allow us to study the behavior of the thyroid as a function of the frequency and estimate the shear wave speed. Since we cannot conclude that the wave from the voice is a plane wave our second concern is the study of the longitudinal section to evaluate displacement through the y-axis.

\section{DATA AVAILABILITY}

The data that support the findings of this study are available from the corresponding author upon reasonable request.

\section{REFERENCES}

${ }^{1}$ H. Monpeyssen, J. Tramalloni, S. Poirée, O. Hélénon, and J.-M. Correas, "Elastography of the thyroid," Diagnostic and interventional imaging 9 (2013), 10.1016/j.diii.2013.01.023.

${ }^{2}$ R. Rahbari, L. Zhang, and E. Kebebew, "Thyroid cancer gender disparity," Future oncology (London, England) 6, 1771-9 (2010).

${ }^{3}$ L.-J. Liao, H.-W. Chen, W.-L. Hsu, and Y.-S. Chen, "Comparison of strain elastography, shear wave elastography, and conventional ultrasound in diagnosing thyroid nodules," Journal of Medical Ultrasound 27, 26-32 (2019).
${ }^{4}$ S. Dudea and C. Botar Jid, "Ultrasound elastography in thyroid disease," Medical ultrasonography 17, 74-96 (2015).

${ }^{5} \mathrm{M}$. Dighe, D. Hippe, and J. Thiel, "Artifacts in shear wave elastography images of thyroid nodules," Ultrasound in Medicine \& Biology 44 (2018), 10.1016/j.ultrasmedbio.2018.02.007

${ }^{6} \mathrm{E}$. Fleury, "Elastography for the evaluation of thyroid nodules in pediatric patients," Radiologia Brasileira 52 (2019), 10.1590/0100-3984.2018.0034.

U. Bae, M. Dighe, V. Shamdasani, S. Minoshima, T. Dubinsky, and Y. Kim, "6f-6 thyroid elastography using carotid artery pulsation: A feasibility study," Proceedings of the IEEE Ultrasonics Symposium , 614-617 (2006).

${ }^{8}$ T. Gallot, S. Catheline, P. Roux, J. Brum, N. Benech, and C. Negriera, "Passive elastography: Shear-wave tomography from physiological-noise correlation in soft tissues," Ultrasonics, Ferroelectrics and Frequency Control, IEEE Transactions on 58, $1122-1126$ (2011).

K. Aki and B. Chouet, "Origin of coda waves: source, attenuation and scattering effects," Journal of Geophysical Research: Solid Earth 80, 3322-3342 (1975).

${ }^{10} \mathrm{M}$. Campillo and A. Paul, "Long-range correlations in the diffuse seismic coda," Science (New York, N.Y.) 299, 547-9 (2003).

${ }^{11}$ N. Shapiro and M. Campillo, "Emergence of broadband rayleigh waves from correlations of the ambient noise," Geophys. Res. Lett 8180 (2004), 10.1029/2004GL019491.

${ }^{12} \mathrm{M}$. Campillo, "Phase and correlation in 'random' seismic fields and the reconstruction of the green function," Pure and Applied Geophysics 163 475-502 (2006)

${ }^{13}$ A. Hoeks, P. Brands, T. Arts, and R. Reneman, "Subsample volume processing of doppler ultrasound signals," Ultrasound in medicine \& Biology 20, 953-65 (1994)

${ }^{14}$ B. Giammarinaro, Focusing of shear shock waves in incompressible solids, Theses, Université Pierre et Marie Curie - Paris VI (2016).

${ }^{15} \mathrm{E}$. Zabolotskaya, M. Hamilton, Y. Mlinskii, and G. Meegan, "Modeling of nonlinear shear waves in soft solids," Acoustical Society of America Journal 116, 2807-2813 (2004)

${ }^{16}$ P. Badin, G. Bailly, L. Reveret, M. Baciu, C. Segebarth, and C. Savariaux, Three-dimensional linear articulatory modeling of tongue, lips and face, based on mri and video images," Journal of Phonetics 30, 533-553 (2002).

${ }^{17}$ A. Zorgani, R. Souchon, A.-H. Dinh, J. Chapelon, J.-M. Ménager, S. Lounis, $\mathrm{O}$. Rouvière, and S. Catheline, "Brain palpation from physiological vibrations using mri," Proceedings of the National Academy of Sciences of the United States of America 112 (2015), 10.1073/pnas.1509895112.

${ }^{18}$ S. Catheline, "Passive elastography: A seismo-inspired tomography of the human body," Journal of the Acoustical Society of America 140, 3138-3138 (2016).

${ }^{19}$ T. Loupas, J. Powers, and R. Gill, "An axial velocity estimator for ultrasound blood-flow imaging, based on a full evaluation of the doppler equation by means of a 2-dimensional autocorrelation approach," Ultrasonics, Ferroelectrics and Frequency Control, IEEE Transactions on 42, 672 - 688 (1995).

${ }^{20}$ S. Catheline, R. Souchon, M. Rupin, J. Brum, and J. Chapelon, "Tomography from diffuse waves: Passive shear wave imaging using low frame rate scanners," Applied Physics Letters 103 (2013), 10.1063/1.4812515.

${ }^{21}$ M. Tanter and M. Fink, "Ultrafast imaging in biomedical ultrasound," IEEE transactions on ultrasonics, ferroelectrics, and frequency control 61, 102119 (2014)

${ }^{22}$ J. Brum, M. Bernal, J.-L. Gennisson, and M. Tanter, "In vivo evaluation of the elastic anisotropy of the human achilles tendon using shear wave dispersion analysis," Physics in medicine and biology 59, 505-523 (2014).

${ }^{23}$ J.-L. Gennisson, M. Rénier, S. Catheline, C. Barrière, J. Bercoff, M. Tanter, and M. Fink, "Acoustoelasticity in soft solids: Assessment of the nonlinear shear modulus with the acoustic radiation force," The Journal of the Acoustical Society of America 122, 3211-9 (2008). 


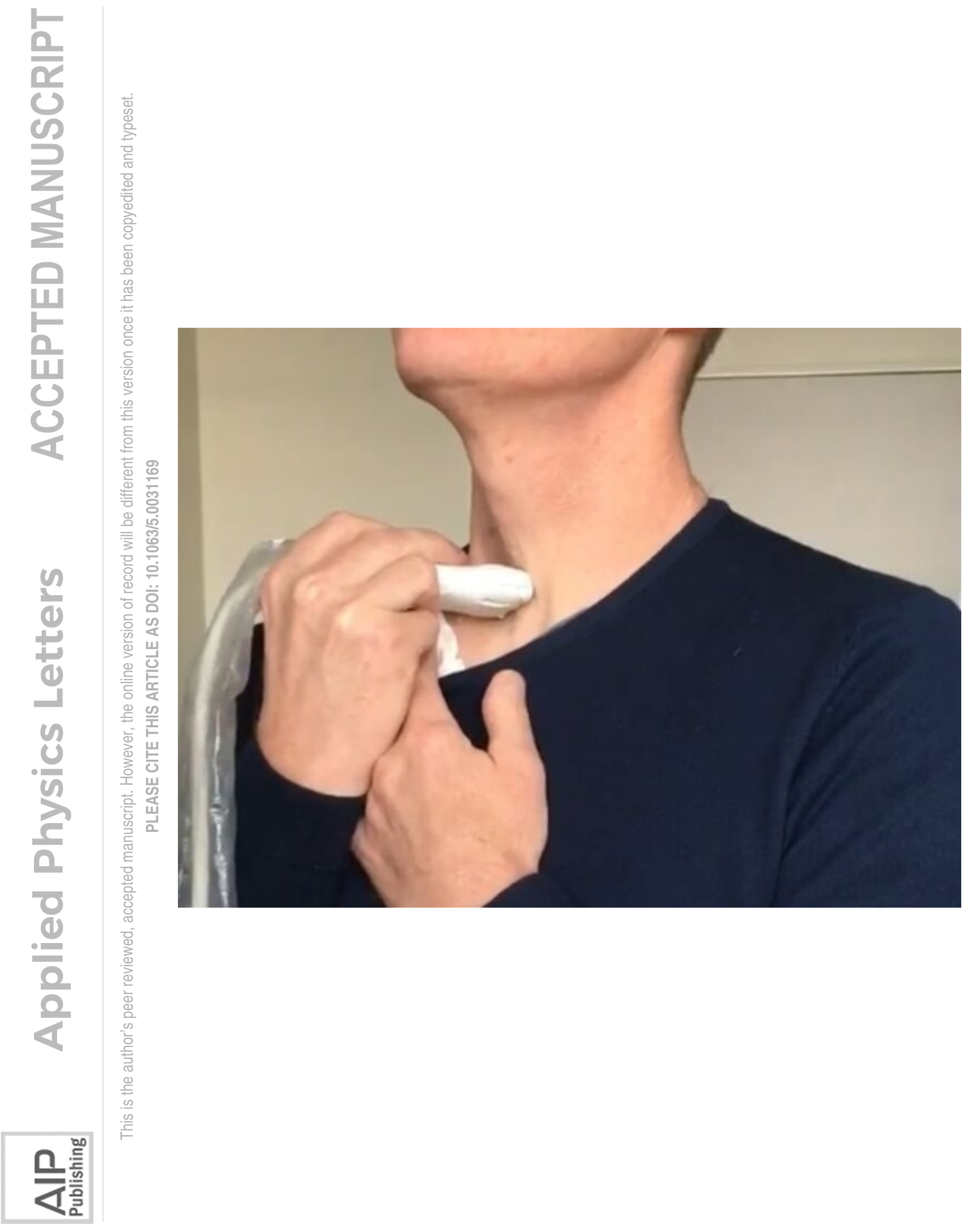




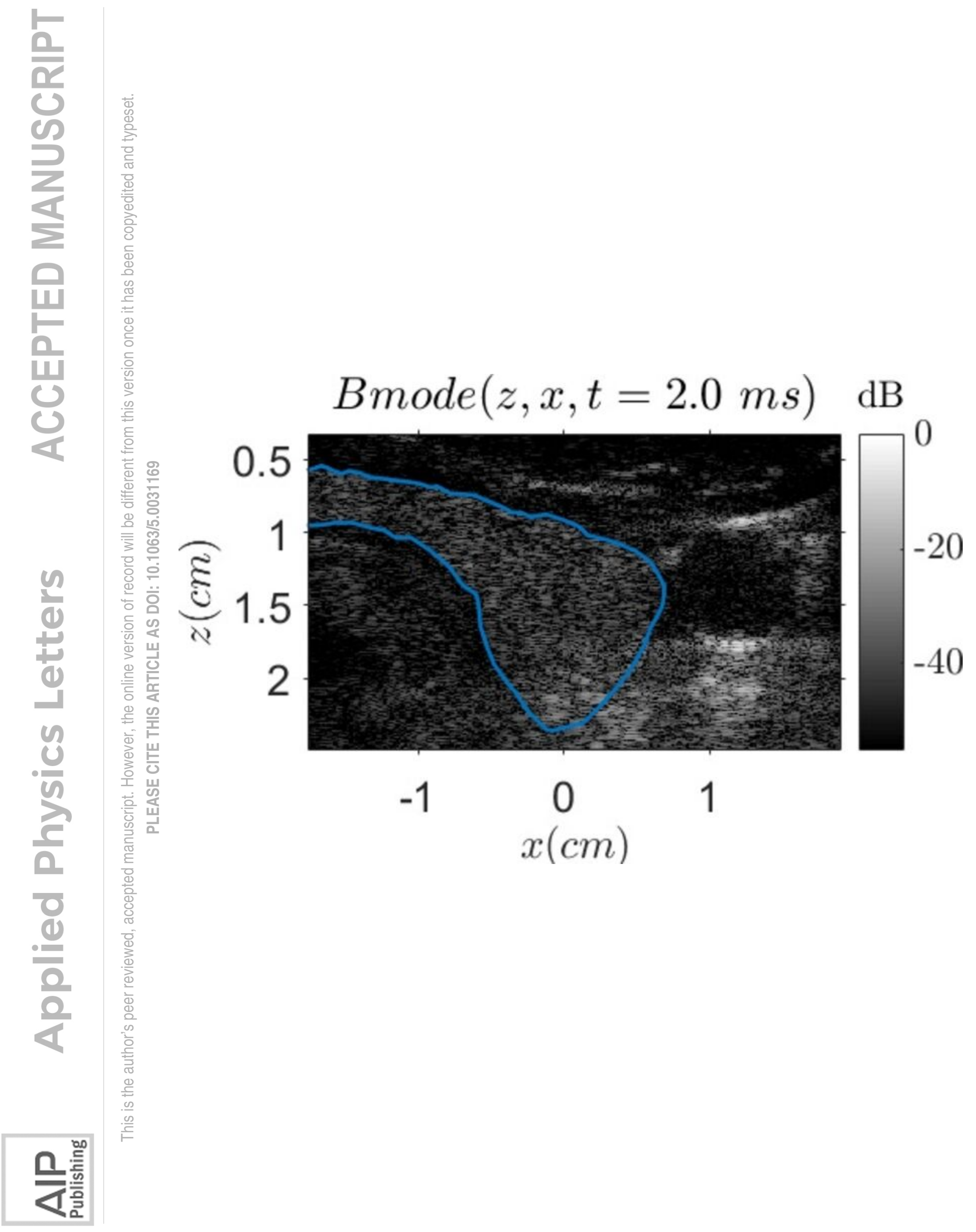




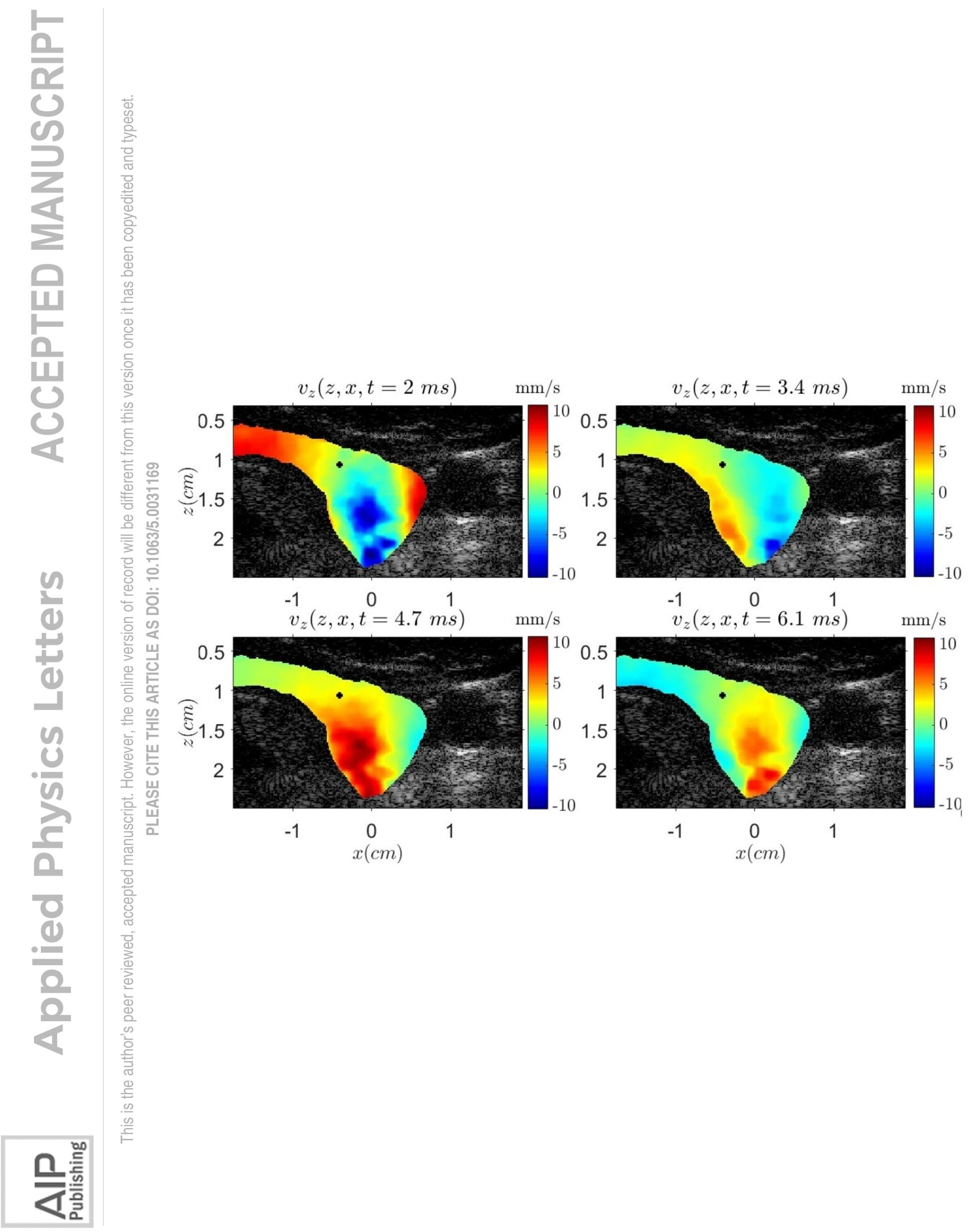




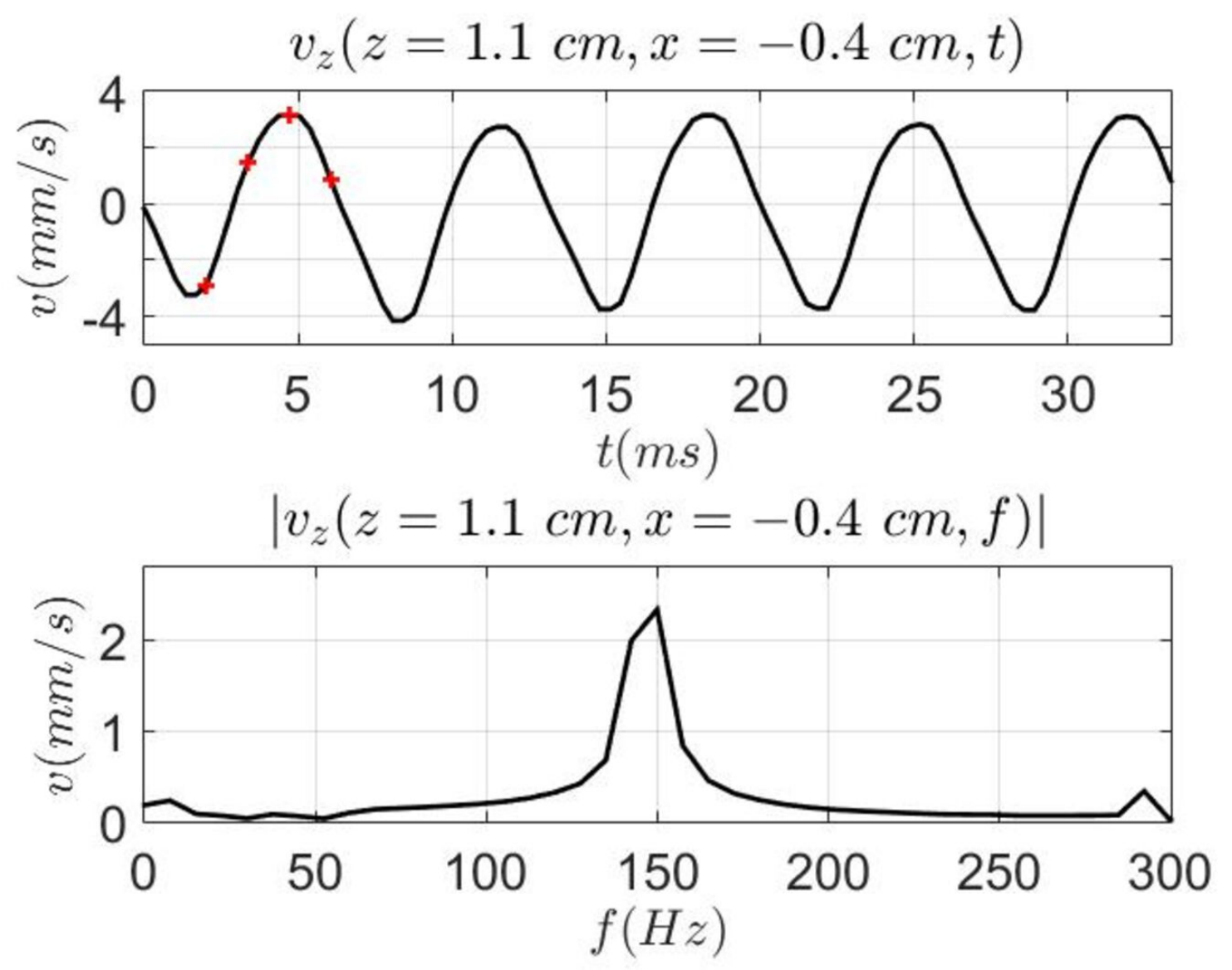

兄旁言 


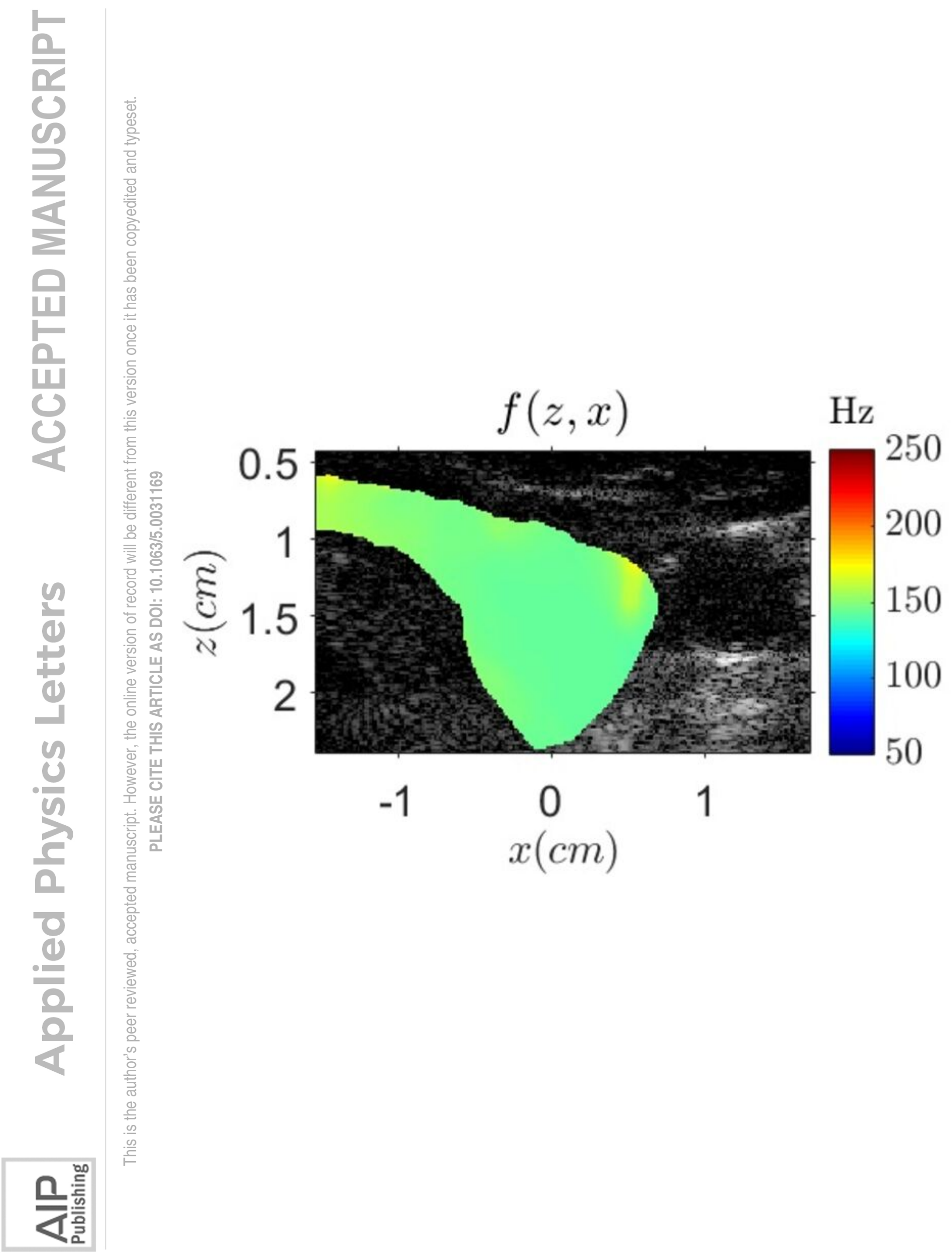




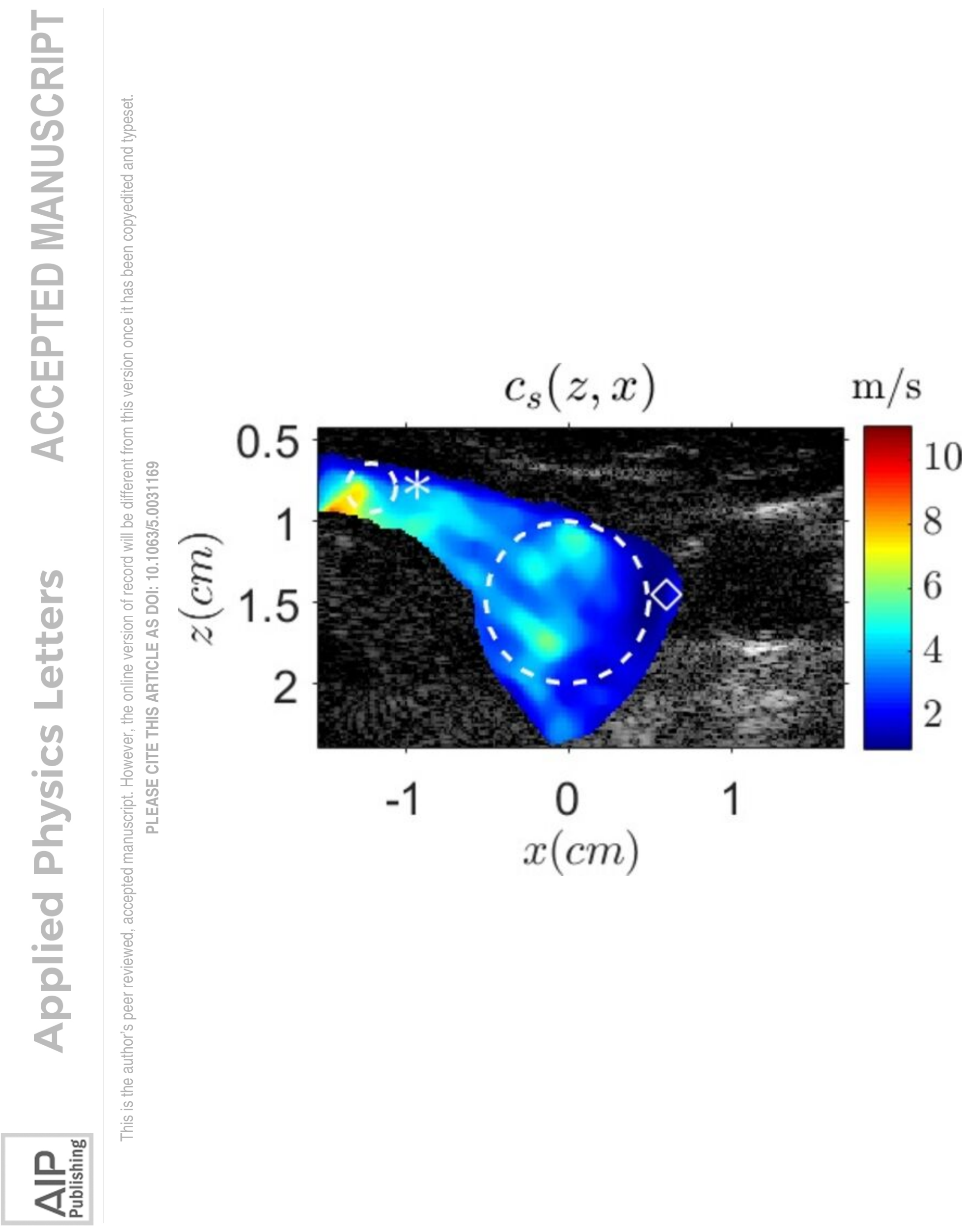




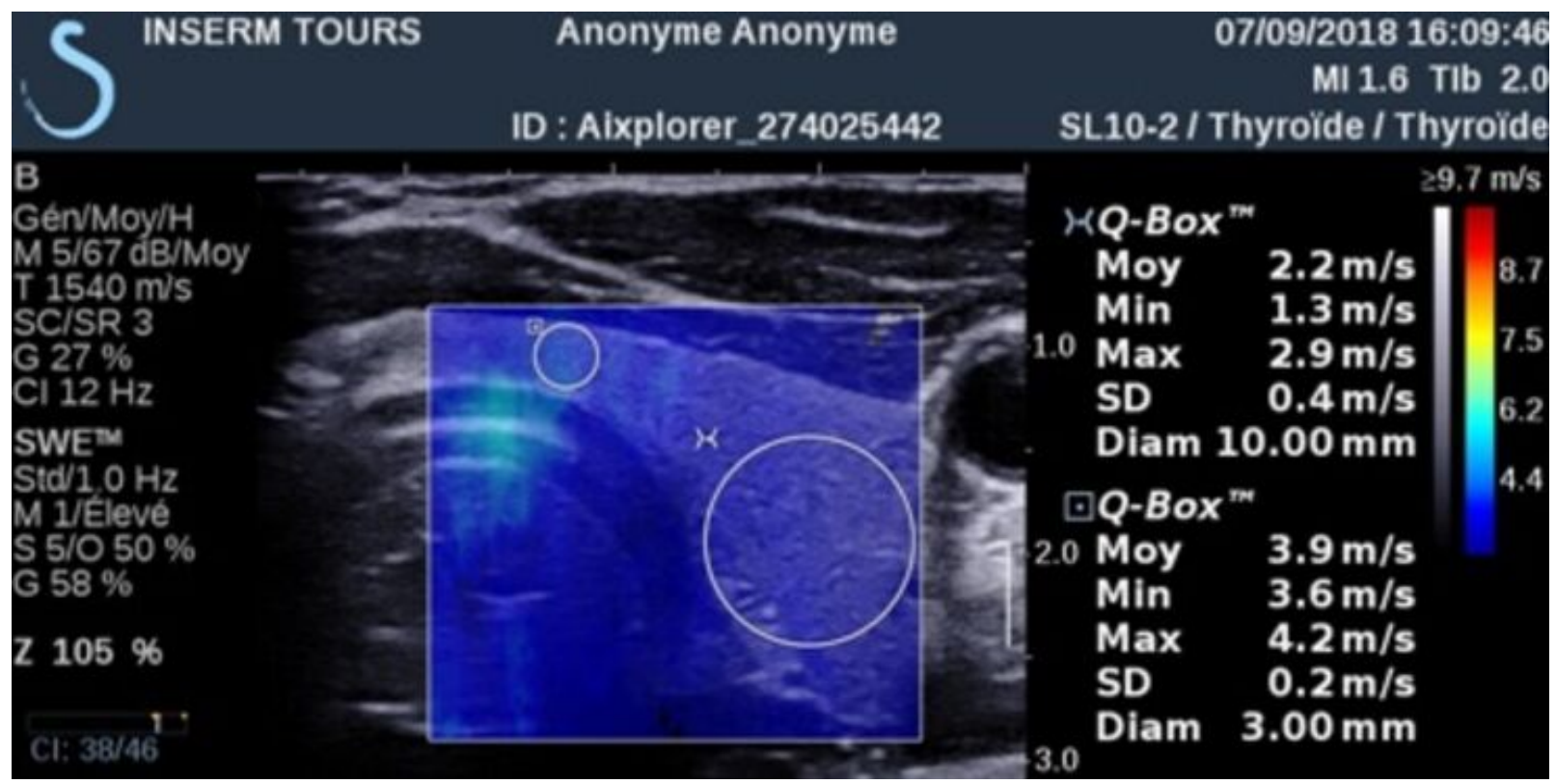

